\title{
Application software for teaching the polarization ellipse
}

\section{Aplicación para la enseñanza de la elipse de polarización}

\author{
Camilo Cano ${ }^{1,}{ }^{*}$, Santiago Echeverri-Chacón ${ }^{2,3}$, Carlos Cuartas-Vélez ${ }^{1}$, Luciano Angel ${ }^{1}$, \\ René Restrepo ${ }^{1}$ \\ 1. Applied Optics Group, Escuela de Ciencias, Universidad EAFIT, Medellín, Colombia \\ 2. Department of Photonics Engineering, Technical University of Denmark, 2800 Kgs. Lyngby, Denmark \\ 3. Mellanox Technologies Denmark ApS, 4000 Roskilde, Denmark \\ ${ }^{(*)}$ E-mail: ccanoba@eafit.edu.co
}

Received: 18/12/2017 Accepted 06/07/2018

DOI: $10.7149 /$ OPA.51.3.50307

\begin{abstract}
:
This paper describes the development of a computational application that calculates the configuration of a polarization state generator, according to the parameters of a desired polarization ellipse. The most general way to describe the polarization state of light is an ellipse, however, it is rarely used in practical courses in which applications are limited to the case of linear or circular polarization. To generate a desired polarization ellipse, a polarizer and a pair of retarders of $\lambda / 2$ and $\lambda / 4$ can be used, the problem is to determine the angle of rotation of the retarders. We present an open source algorithm to calculate these angles by using a minimization method applied to the Jones calculus description of polarizing elements. This development aims to help research and education activities to promote a more practical understanding of the polarimetric properties of light.
\end{abstract}

Key words: Polarization ellipse; Jones calculus; Minimization.

\section{RESUMEN:}

En este trabajo se presenta el desarrollo de una aplicación computacional que permite determinar la configuración de un generador de estados de polarización en función de los parámetros de una elipse de polarización deseada. La manera más general de describir la polarización de la luz es una elipse, sin embargo, este estado rara vez se aborda en un curso práctico en los cuales las aplicaciones se limitan al caso de la polarización lineal o circular. Para generar una elipse de polarización deseada se puede utilizar un polarizador y un par de retardadores de $\lambda / 2$ y $\lambda / 4$, el problema está en determinar a qué ángulo deben estar ubicados los retardadores. Se presenta un algoritmo de acceso libre que calcula estos ángulos mediante un método de minimización aplicado al cálculo de Jones. Este desarrollo va dirigido a actividades de investigación y educación, para promover una comprensión práctica de las propiedades polarimétricas de la luz.

Palabras clave: Elipse de polarización; Cálculo de Jones; Minimización.

\section{REFERENCES AND LINKS / REFERENCIAS Y ENLACES}

[1] G. Duree, Optics for Dummies. Indianapolis, John Wiley \& Sons (2011).

[2] E. Hecht, Óptica. Madrid, Pearson Educación (2000).

[3] E. Wolf, Progress in Optics. New York, Elsevier (2000).

[4] G. Brooker, Modern Classical Optics. New York, OUP Oxford (2003).

[5] G. Gurin, K. Titov, Y Ilyin, A. Tarasov, "Induced polarization of disseminated electronically conductive minerals: a semi-empirical model”, Geophys. J. Int. 200, 1555-1565 (2015).

[6] C. Bérubé, M. Chouteau, P. Shamsipour, R. Enkin, G. Olivo, "Bayesian inference of spectral induced polarization parameters for laboratory complex resistivity measurements of rocks and soils", Comput. Geosci. 105, 51-64 (2017). 
[7] F. Fontaine, G. Barruol, B. Kennett, G. Bokelmann, D. Reymond, "Upper mantle anisotropy beneath Australia and Tahiti from P wave polarization: Implications for real-time earthquake location", J. Geophys. Res. 114, B3 (2009).

[8] H. Sarkissian, S. Serak, N. Tabiryan, L. Glebov, V. Rotar, B. Zeldovich, "Polarization-controlled switching between diffraction orders in transverse-periodically aligned nematic liquid crystals”, Opt. Lett. 31, 2248-2250 (2006).

[9] A. Golaraei, L. Kontenis, R. Cisek, D. Tokarz, S. Done, B. Wilson, V. Barzda, "Changes of collagen ultrastructure in breast cancer tissue determined by second-harmonic generation double StokesMueller polarimetric microscopy," Biomed. Opt. Express 7, 4054-4068 (2016).

[10] T. Novikova, A. Pierangelo, A. De Martino, A. Benali, P. Validire, "Polarimetric Imaging for Cancer Diagnosis and Staging," Opt. Photonics News 23(10), 26-33 (2012).

[11] A. Yariv, P. Yeh, Optical Waves in Crystals: Propagation and Control of Radiation, New Jersey (2002).

[12] A. Pitilakis, D. Zografopoulos, E. Kriezis, "In-Line Polarization Controller Based on Liquid-Crystal Photonic Crystal Fibers," J. Lightwave Technol. 29, 2560-2569 (2011).

[13] W. Sparks, J. Hough, L. Kolokolova, T. Germer, F. Chen, S. DasSarma, P. DasSarma, F. Robb, N. Manset, I. Reid, F. Macchetto, W. Martin, "Circular polarization in scattered light as a possible biomarker", J. Quant. Spectrosc. Ra. 110, 1771-1779 (2009).

[14] B. Wen, R. Petschek, C. Rosenblatt, "Nematic liquid-crystal polarization gratings by modification of surface alignment," Appl. Opt. 41, 1246-1250 (2002).

[15] E. Collet, Field Guide to Polarization. Bellingham, SPIE Press (2005).

[16] D. Goldstein, Polarized Light, New York. Taylor \& Francis Group (2011).

[17] W. McMaster, “Matrix Representation of Polarization”, Rev. Mod. Phys. 33, 8-28 (1961).

[18] L. Rios, "Derivative-free optimization: a review of algorithms and comparison of software implementations", J. Global Optim. 56, 1247-1293 (2013).

\section{Introduction}

Optics is one of the main branches of physics, and is related to the study of different properties of light and its interaction with matter [1]. Some of the several applications in optics are related to the polarization of light [2-4]. Since its discovery, polarization has become a powerful tool for studying many kinds of minerals [5-7], and nowadays it is used for the evaluation of biologicals tissues, like connective tissue which is rich in collagen, allowing the early identification of diseases like cancer [8-10]. Due to the wide range of polarized light measurement applications, it has become an important subject in several university level courses, not only in physics but in other areas such as geology and biology.

Most polarization measurement techniques make use of an ellipsometer or polarimeter. These scientific instruments allow the measurement of changes in the polarization state of light when it interacts with an optically active substance [11]. Most applications based on polarized light make use of linear and circular polarization states, but the introduction of liquid crystal displays as polarizing tools is increasing the interest of understanding elliptical states [12-14]. In certain polarization experiments, like the characterization of birefringent optical elements, the generation and analysis of large sets of elliptical polarization states is required. In practice, this is done using optical elements, such as polarizers and phase retarders that modify a known polarization state.

When dealing with linear or circular polarization, it is relatively easy to discern how to use retarders and polarizers to produce a specific polarization state. There is however, a big chance that even experts find some difficulties at generating non-standard polarization states from their mathematical representation. While performing ellipsometry measurements we identified the need to easily translate book definitions of polarization states into positions of the optical elements that generate these states. For instance, consider that you have to measure an undesired change in polarization introduced by a component already characterized, however, it is impossible to experimentally compensate it on the set-up as its description in terms of polarizers and retarders is unknown.

In this work, we present an intuitive and straightforward python-based graphical application built to quickly translate polarization states into angles of physical elements for use in teaching and research environments. Some of the more widely known notations for polarization states were used as input in the 
software, those are: polarization ellipse parameters and the Dirac notation, which have a direct conversion to Jones calculus and the Poincare sphere [1].

\section{Ellipse of polarization and its representation}

In the electromagnetic theory of light, optical fields are represented as waves that propagate in empty space. A beam of light can be defined either by its electric or magnetic field, which are periodic and orthogonal between them [11]. Since the magnitude of electric fields is considerably larger than the magnetic fields, the first is usually used to describe the behavior of light in a simple way. According to Fresnel's theory, the electric field of a beam can be expressed as a pair of sinusoidal oscillations $E_{x}(z, t)$ in the plane $x-z$ and $E_{y}(z, t)$ in $y-z[15]$, given by

$$
\begin{aligned}
& E_{x}(z, t)=E_{0 x} \cos \left(\omega t-k z+\delta_{x}\right), \\
& E_{y}(z, t)=E_{0 y} \cos \left(\omega t-k z+\delta_{y}\right),
\end{aligned}
$$

where $\omega$ is the angular frequency, $t$ is the time, $k$ is the wave number and $z$ is the position in the propagation axis. All those describe the phase of the wave that is expressed as $\sigma=\omega t-k z . E_{0 x}$ and $E_{0 y}$ are the maximum amplitudes of the field in $x$ and $y ; \delta_{x}$ and $\delta_{y}$ are the initial phases. Applying trigonometric identities, Eq. (1a) and Eq. (1b) can be rewritten as:

$$
\begin{aligned}
& \frac{E_{x}(z, t)}{E_{0 x}}=\cos (\sigma) \cos \left(\delta_{x}\right)-\sin (\sigma) \sin \left(\delta_{x}\right), \\
& \frac{E_{y}(z, t)}{E_{0 y}}=\cos (\sigma) \cos \left(\delta_{y}\right)-\sin (\sigma) \sin \left(\delta_{y}\right) .
\end{aligned}
$$

Multiplying Eq. (2a) and Eq. (2b) by $\sin \left(\delta_{y}\right)$ and $\sin \left(\delta_{x}\right)$ respectively and subtracting them we get

$$
\frac{E_{x}(z, t)}{E_{0 x}} \sin \left(\delta_{y}\right)-\frac{E_{y}(z, t)}{E_{0 y}} \sin \left(\delta_{x}\right)=\cos (\sigma) \sin \left(\delta_{y}-\delta_{x}\right),
$$

repeating the procedure using $\cos \left(\delta_{y}\right)$ and $\cos \left(\delta_{x}\right)$ a second expression is obtained

$$
\frac{E_{x}(z, t)}{E_{0 x}} \cos \left(\delta_{y}\right)-\frac{E_{y}(z, t)}{E_{0 y}} \cos \left(\delta_{x}\right)=\sin (\sigma) \sin \left(\delta_{y}-\delta_{x}\right) .
$$

The sum of the square of Eq. (3a) and Eq. (3b) leads to

$$
\frac{E_{x}^{2}(z, t)}{E_{0 x}^{2}}+\frac{E_{y}^{2}(z, t)}{E_{0 y}^{2}}-2 \frac{E_{x}(z, t) E_{y}(z, t)}{E_{0 x} E_{0 y}} \cos (\delta)=\sin ^{2}(\delta),
$$

where $\delta=\delta_{y}-\delta_{x}$ is the relative phase shift between components of the electric field. Equation (4) corresponds to an ellipse equation which means that at a fixed point $z$ the electric field will describe an ellipse like the depicted in Fig. 1 as the time advances [15]. 


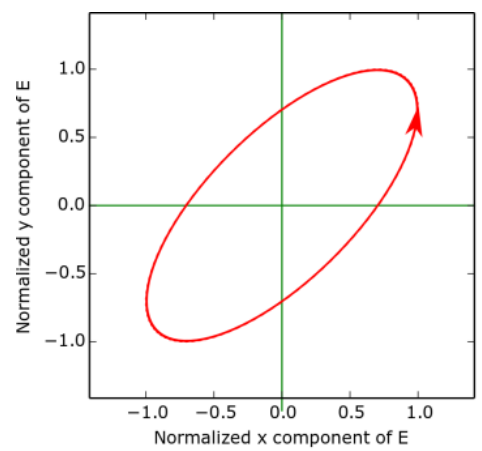

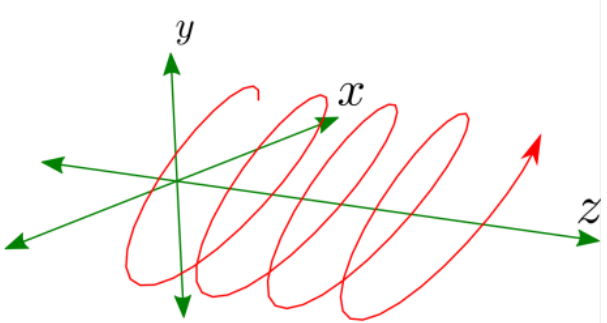

(b)

(a)

Fig. 1. Electric field of a beam with elliptical polarization observed (a) at a fixed point $\mathrm{z}$ and (b) at a fixed time $t$.

Simplifying the description of polarization ellipses into a geometrical definition, a pair of angular parameters are used: the orientation angle $\psi(0 \leq \psi \leq \pi)$ which is the angle between the larger semi-axis of the ellipse and the $x$ axis, and the ellipticity angle $\chi(-\pi / 4 \leq \chi \leq \pi / 4)[11,15]$. Figure 2 presents these parameters.

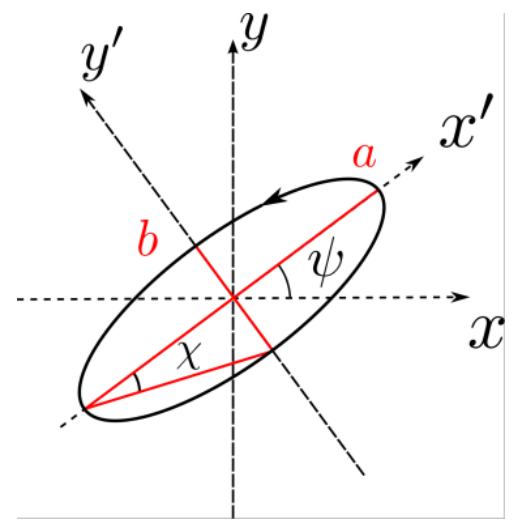

Fig. 2. Polarization ellipse and its angular parameters

Although Eq. (4) is the most general representation to describe the polarization state of light, there are some cases known as degenerate polarization states, which are used in most situations, and frequently taught in optics courses. Those cases are obtained for a particular set of values of $E_{0 x}, E_{0 y}$ and $\delta$ [16]. Degenerate states of polarization can be contained in three instances:

$E_{0 y}=0$. In this case $E_{y}(z, t)$ is zero, so that the field will be described by Eq. (1a), indicating only oscillation of the electric field in the direction of the $x$ axis. Thereby, is said that the light is linearly polarized in the $x$ direction, or horizontally polarized. Equivalently, if $E_{0 y}=0$ and $E_{y}(z, t) \neq 0$ there would be oscillation only on the $y$-axis, and the light is linearly polarized in $y$, or vertically polarized.

- $\delta=0$ or $n \pi$, Eq. (4) is simplified to:

$$
\frac{E_{x}^{2}}{E_{0 x}^{2}}+\frac{E_{y}^{2}}{E_{0 y}^{2}} \pm 2 \frac{E_{x} E_{y}}{E_{0 x} E_{0 y}}=0 .
$$

Isolating $E_{y}$ from Eq. (5a) we obtain

$$
E_{y}= \pm \frac{E_{0 x}}{E_{0 y}}=E_{x} .
$$


Equation (5b) corresponds to a straight line with slope $\pm E_{0 x} / E_{0 y}$ and intercept in 0 . In this case, the light is linearly polarized with an angle of $\pm E_{0 x} / E_{0 y}$ (rad). For a value of $\delta=0$ the slope is negative, while positive for $\delta=\pi$. If $E_{0 x}=E_{0 y}$, then $E_{y}= \pm E_{x}$. If the slope is positive, light is linearly polarized at $+45^{\circ}$, and in the opposite case, light is linearly polarized at $-45^{\circ}$.

- $\quad$ If $E_{0 x}=E_{0 y}$ And $\delta=(2 n-1) \pi / 2$, Eq. (4) is reduced to

$$
\frac{E_{x}^{2}}{E_{0}^{2}}+\frac{E_{y}^{2}}{E_{0}^{2}}=1 \text {. }
$$

Equation (6) describes a circle. In this case light has right circular polarization for $\delta=\pi / 2$ or left circular polarization for $\delta=3 \pi / 2$.

The graphical representation of the degenerate polarization states is presented in Fig. 3.

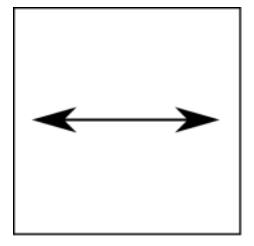

LHP: $\mathrm{E}_{0 \mathrm{x}}=0$

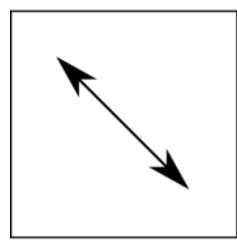

$\mathrm{L}-45 \mathrm{P}$

$\mathrm{E}_{0 \mathrm{y}}=\mathrm{E}_{0 \mathrm{x}}, \quad \delta=\pi$

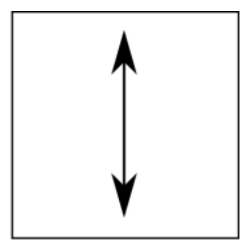

LVP: $\mathrm{E}_{0 \mathrm{y}}=0$

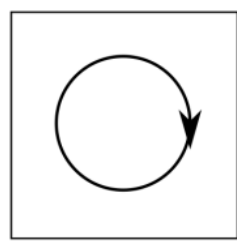

$\mathrm{RCP}:$ $\mathrm{E}_{0 \mathrm{y}}=\mathrm{E}_{0 \mathrm{x}}, \dot{\delta}=\pi / 2$

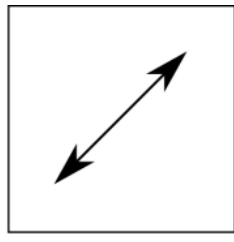

L+45P:
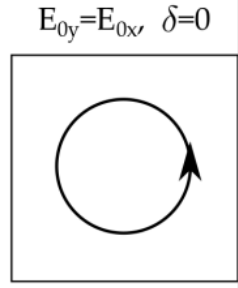

LCP:

$\mathrm{E}_{0 \mathrm{y}}=\mathrm{E}_{0 \mathrm{x}}, \dot{\delta}=-\pi / 2$

Fig. 3. Degenerate polarization states. LHP: Linear Horizontal Polarization; LVP: Linear Vertical Polarization; L+45P: Linear +45 Polarization; L-45P: Linear -45 Polarization; RCP: Right Circular Polarization and LCP: Left Circular Polarization.

\section{2.a. Jones Calculus}

Jones calculus is a matrix formulation used to describe the polarization state of light and polarizing components. In Jones formulation, light polarization is expressed by a $2 \times 1$ vector called Jones vector, where the first component corresponds to the electric field in the $x$ axis and the second one the field in the $y$ axis [15]. The Jones vector for a field $\vec{E}$ is given by

$$
\vec{E}=\left(\begin{array}{l}
E_{x} \\
E_{y}
\end{array}\right)=\left(\begin{array}{l}
E_{0 x} e^{i \delta_{x}} \\
E_{0 y} e^{i \delta_{y}}
\end{array}\right) .
$$

Polarizing components are represented by $2 \times 2$ matrices which operates on the Jones vectors, and generating a new output vector. In this way, it is possible to determine the emerging polarization state from a polarizing element for a given input. Some of the most used polarizing components are linear polarizers, half-wave plate retarders and quarter-wave plate retarders $[15,16]$. Those are described by the following matrices:

$$
J_{L H P}=\left(\begin{array}{ll}
1 & 0 \\
0 & 0
\end{array}\right), \quad J_{H W P}=\left(\begin{array}{cc}
e^{i(\pi / 2)} & 0 \\
0 & e^{-i(\pi / 2)}
\end{array}\right), \quad J_{Q W P}=\left(\begin{array}{cc}
e^{i(\pi / 4)} & 0 \\
0 & e^{-i(\pi / 4)}
\end{array}\right),
$$


where $J_{L H P}$ is the Jones matrix of an ideal linear horizontal polarizer, $J_{H W P}$ and $J_{Q W P}$ are a half- and quarterwave plates respectively. Polarizing elements can be oriented in different directions using the rotation Jones matrix $R(\theta)$ [15]

$$
R(\theta)=\left(\begin{array}{cc}
\cos (\theta) & \sin (\theta) \\
-\sin (\theta) & \cos (\theta)
\end{array}\right)
$$

Any polarimetric system can be modeled as the product of the different components that conform it. Particularly, when the input light is polarized (i.e. laser source), a polarization state generator (PSG) with the least energy loss constituted of retarders is defined as

$$
\vec{E}_{\text {out }}=R^{T}\left(\theta_{2}\right) J_{Q W P} R\left(\theta_{2}\right) R^{T}\left(\theta_{1}\right) J_{H W P} R\left(\theta_{1}\right) \vec{E}_{\text {in }}
$$

where $\vec{E}_{\text {in }}$ is a known polarization state, usually linear polarization in $x$ or $y \cdot \vec{E}_{\text {out }}$ is the output polarization state obtained with the PSG, $\theta_{1}$ is the angle at which the half-wave plate retarder should be oriented and $\theta_{2}$ the quarter-wave plate angle.

Another way to represent polarization states based on Jones calculus, is the Dirac notation. By making use of quantum mechanics notation, it is possible to describe polarization states with a couple of parameters: the phase shift between component $\delta$ and an angular factor $\alpha=\operatorname{atan}\left(E_{0 x} / E_{0 y}\right)$ [17]. This way a polarization state can be describe under Bra-Kets notation as $|\alpha, \delta\rangle$ [17].

Polarization states can also be expressed in the Poincare sphere, where the Stokes parameters are plotted, neglecting the first component $\left(S_{0}\right)$ [15]. However, we focused our application to the aforementioned notations.

\section{Calculation of PSG configuration for a desired ellipse}

Suppose you are given the task to experimentally generate a linear state of polarization defined by any of the following descriptions (Fig. 4).

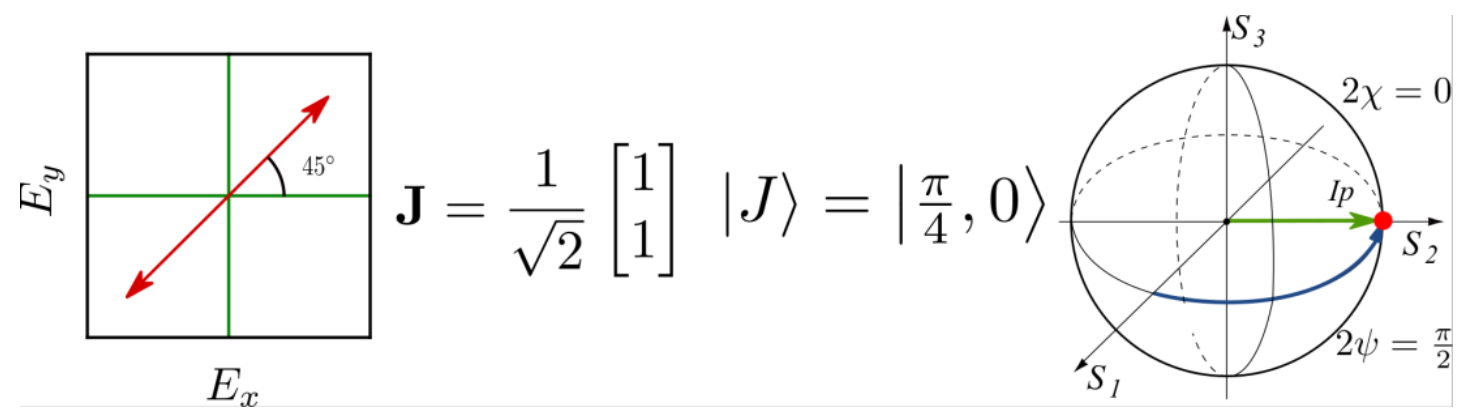

Fig. 4. Simple polarization state in its graphical representation, Jones, Dirac and Poincare notation (left to right).

This is an easy task because it is one of the most basic states of polarization. We could either: a) use a $\lambda / 2$ retarder at an angle of $22.5^{\circ}$ with respect to horizontal polarization, or b) locate a polarizer at an angle of $45^{\circ}$ along a circularly polarized beam. However, if you are supposed to generate uncommon polarization states, such as the one defined below in Fig. 5., you may encounter great difficulties in any definition you decide to follow. 


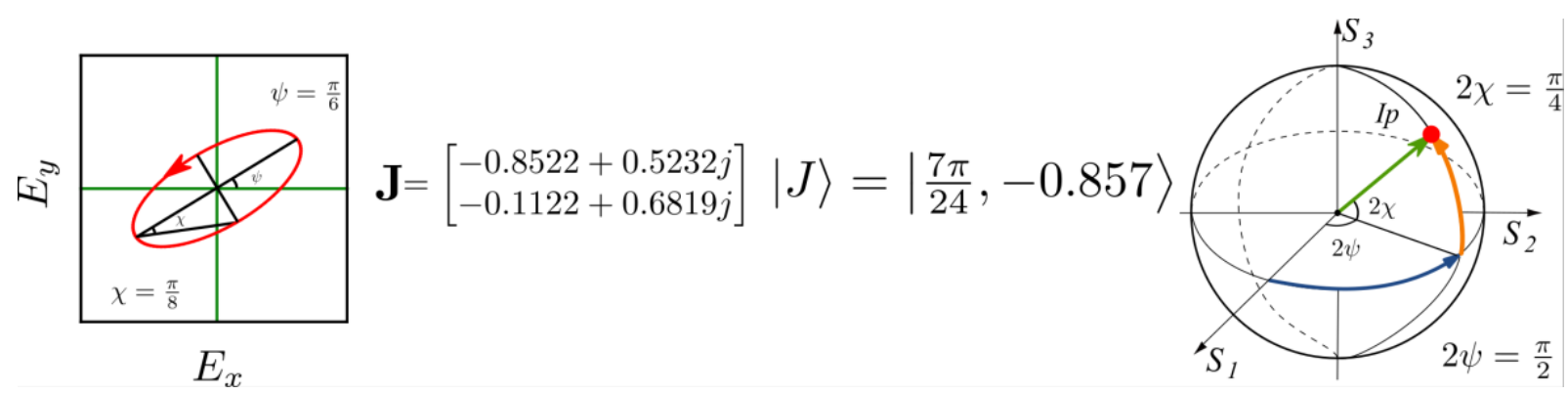

Fig. 5. Elliptical polarization state in its graphical representation, Jones, Dirac and Poincare notation (left to right).

Even if you are an expert in polarimetry, you will have a tedious time trying to figure out the configuration in which you must set $\lambda / 4$ and $\lambda / 2$ retarders to generate this state. In order to solve this problem, we developed an easy to use application to translate complex polarization states into angles of laboratory optical elements that create them. This can be useful for educational and research purposes.

\section{3.a. Transformation of polarization states among notations}

The state of polarization of light can be parameterized in different ways, by using ellipse parameters and matrix expressions such as Jones or Stokes calculus and Dirac notation [15,17]. There are advantages and disadvantages on each of them, for instance, the ease of visualization, compactness, and difficulty for performing calculations. When using coherent light, optical elements in the laboratory are best represented by Jones Matrices acting on Jones vectors. However, there is not a straightforward relation between representations of polarization states and the position of the retarders that generate a state. Instead, it is possible to simulate the optical elements by using Jones matrices, propagating a known Jones vector, and then see if the vector representations are equivalent. There are analytical transformations between representations and most of them are bidirectional as presented in Fig. 6, with the exception of general Jones vectors which have more variables [15,17]. In order to cope with this limitation, we use the minimization procedure depicted in Fig. 6 and explained below.
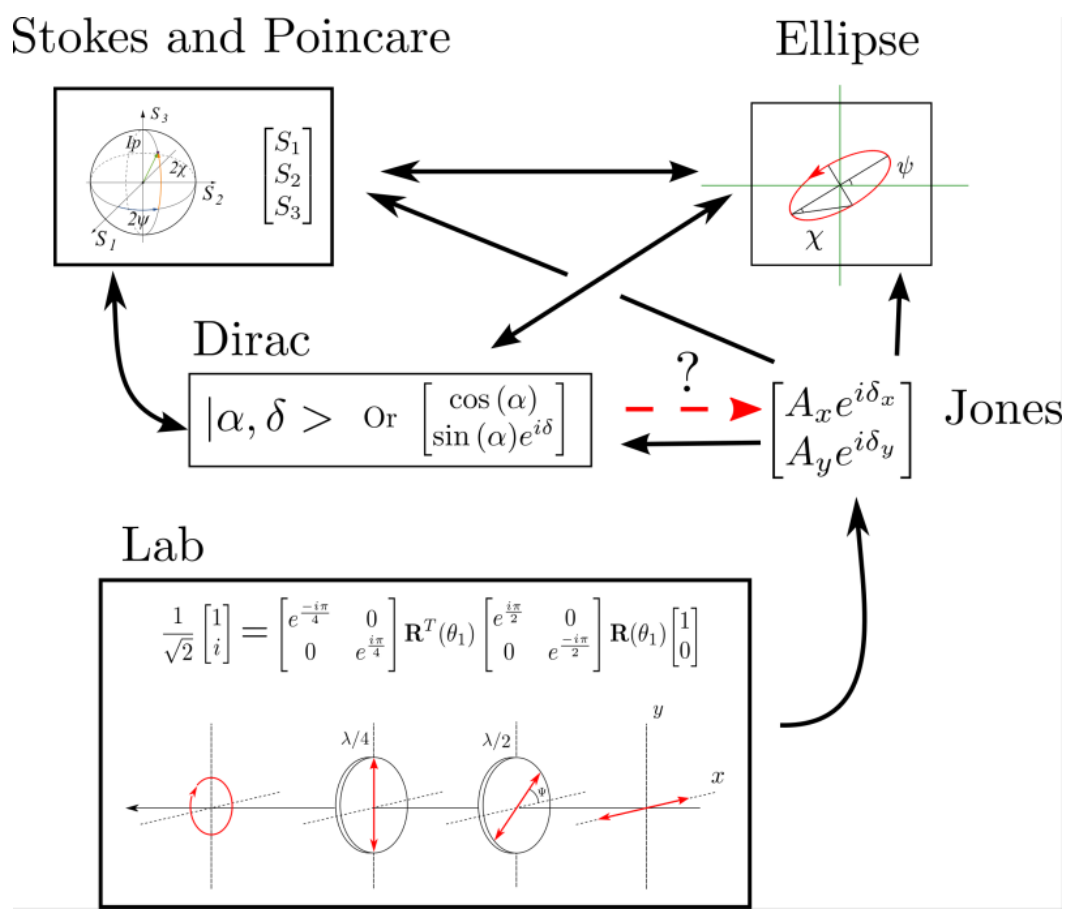

Fig. 6. Conversion between different representation of polarization state. 


\section{3.b. Minimization Procedure}

We implemented a minimization procedure that uses matrix multiplication operations based on Jones vector calculus to find the angles of rotation for the components of a PSG. First, the user introduces a desired ellipse, either by its angular parameters $(\psi, \chi)$ or using Dirac notation $(\alpha, \delta)$. An initial guess is proposed for the angle orientation $\left(\theta_{1}, \theta_{2}\right)$ of the elements, and is used as seed for the minimization procedure, propagating a known input state (LHP) through the described PSG in Eq. (10). Then, the application transforms the resulting Jones vector into Dirac and ellipse angular parameters notation for comparison with the input ellipse. A merit function is used to determine whether the calculated ellipse corresponds to the user request, and the application displays the elements angles and plots the desired ellipse if the error is below $10^{-5}$. If the error does not satisfy the minimization criteria (minimum error), a new set of angles is propose based on a gradient minimization algorithm [18]. In Fig. 7. a flow chart illustrates the process of the algorithm.

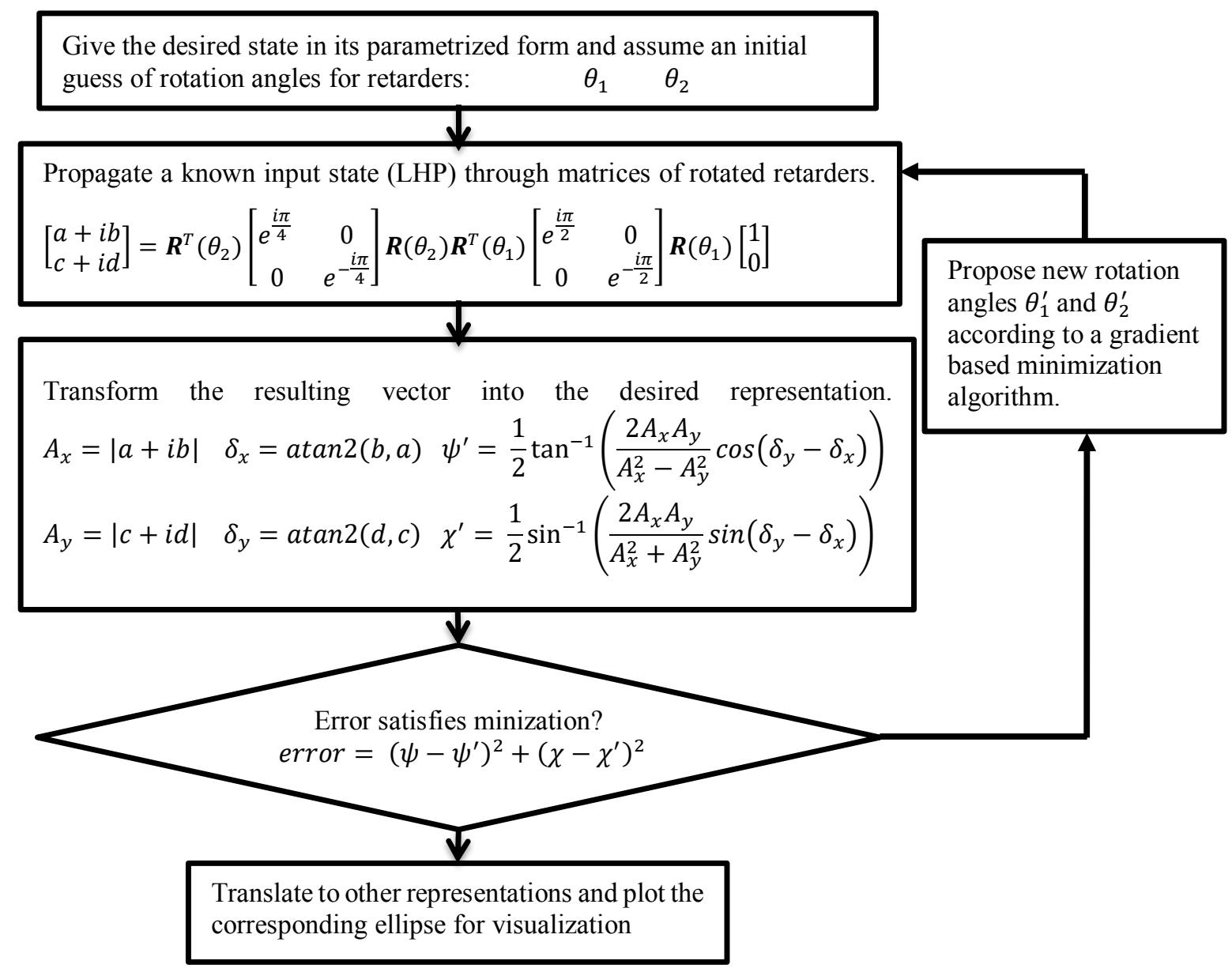

Fig. 7. Flow chart for minimization algorithm for polarizing elements angles orientation.

\section{3.c. Graphical interface}

The result of this work is a Python based interactive application that runs under the Qt4 graphical interface. It is a simple but intuitive platform, in which you can visualize and translate states of polarization defined from three different representations: Dirac notation, angular ellipse parameters and polarizing elements angle. On the bottom right panel, the ellipse direction and ellipticity angle are set, and then translate those parameters to the Dirac and Lab representations. This will automatically plot the trajectory of the pointing vector from a frontal and isometric perspective, allowing the user to view the polarization ellipse and sense of rotation for non-linear states. On the bottom left panel you can give the state in terms of Dirac brakets 
and then translate it to ellipse angles. This implementation is open source and can be found in the following GIT repository: https://github.com/bebopsan/Ellipsometry for dummies

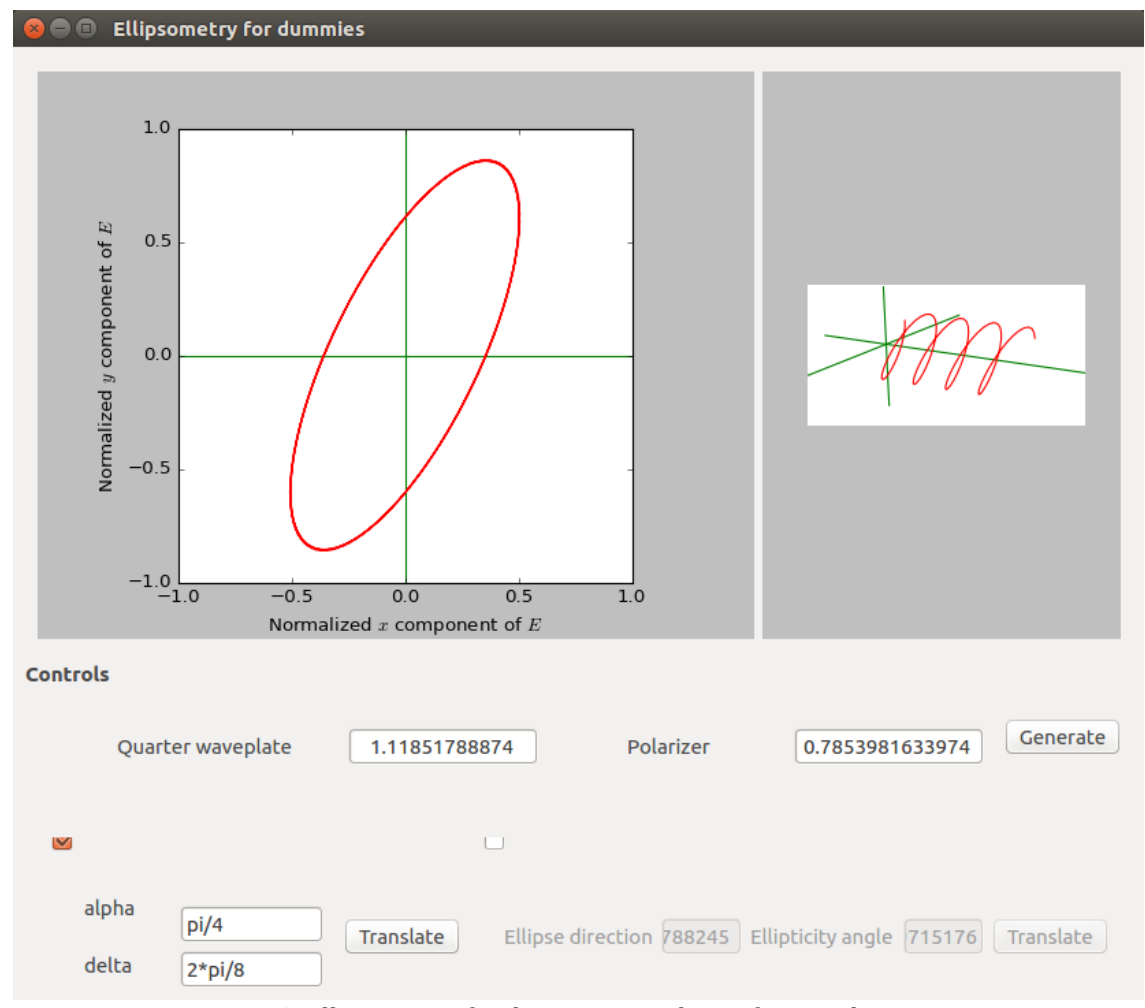

Fig. 8. Ellipsometry for dummies. Final interface in Ubuntu.

\section{Conclusions}

In conclusion, we designed and implemented a software tool for the identification of angles of rotation of optical elements in order to produce a desired polarization state when horizontal polarization is used as input. The procedure consists of a minimization routine involving Jones calculus and well-known analytical transformations. This method allows a fast and easy transition among notations in a simple way, facilitating teaching and research activities. Since we only analyze completely polarized states, there is no need to use Stokes notation, but further implementations are possible with a generalization where depolarization and diattenuation effects are taken into account.

The process of translating polarization states, and finding the angles of optical elements, as well as a graphical representation, was made available to the general public through an open source application based on the Python programming language.

\section{Acknowledgements}

The authors acknowledge to Universidad EAFIT for the funding and support of this project through grant (767-000063), COLCIENCIAS (761). 